\title{
Shared neural mechanisms of visual perception and imagery
}

Nadine Dijkstra ${ }^{1}$, Sander E. Bosch ${ }^{1}$ and Marcel A. J. van Gerven ${ }^{1}$

1. Donders Institute for Brain, Cognition and Behaviour, Radboud University, Nijmegen, the Netherlands

Correspondence: n.dijkstra@donders.ru.nl (N. Dijkstra)

Keywords: mental imagery - neural representations - connectivity - temporal dynamics generative perception - working memory

For decades, the extent to which visual imagery relies on similar neural mechanisms as visual perception has been a topic of debate. Here, we review recent neuroimaging studies comparing these two forms of visual experience. Their results suggest that there is large overlap in neural processing during perception and imagery: neural representations of imagined and perceived stimuli are similar in visual, parietal and frontal cortex. Furthermore, perception and imagery seem to rely on similar top-down connectivity. The most prominent difference is the absence of bottom-up processing during imagery. These findings fit well with the idea that imagery and perception rely on similar emulation or prediction processes.

\section{Externally and internally generated visual experience}

A large part of our sensory experience is visual. When walking down the street, we are bombarded with different colors, shapes and textures. Also when thinking about future or past events, most people tend to experience a rapid stream of detailed images [1]. Visual experience can be triggered externally, by events in the outside world that change the light that falls unto our retinas, such as during perception (see Glossary); or internally, by information from memory via a process known as mental imagery (see Box 1 on the relationship between imagery and working memory). Generally, these are seen as two distinct phenomena. However, they are phenomenologically similar which can sometimes lead us to question whether we really saw something or whether it was just our imagination.

The question to what extent visual imagery relies on the same neural mechanisms as perception has been a topic of debate for decades. Originally, the debate was centered around the question whether imagery, like perception, relied on depictive, picture-like representations, or on symbolic, language-like representations [2-5]. Due to imagery's 
inherently private nature, for a long time it was impossible to address this question. Neuroimaging studies on the involvement of the primary visual cortex during imagery have now largely resolved this debate in favor of the depictive view [6]. However, a broader perspective, addressing the involvement and interaction of brain regions beyond the primary visual cortex, has been missing.

The current review explores to what extent externally and internally generated visual experiences rely on similar neural mechanisms. We discuss the findings with respect to visual areas which are important in the depictivism-versus-symbolism debate, but we also focus on the involvement of parietal and frontal areas. Next, we focus on the temporal dynamics of neural processing during both forms of visual experience. After that, we discuss the overlap in directional connectivity between perception and imagery. We finish by concluding that perception and imagery are in fact highly similar and we discuss the issues and questions raised by this conclusion. 


\section{Box 1: Visual Imagery and Working Memory}

It has been suggested that imagery and working memory are one and the same [93]. There is overlap in neural representations between imagined stimuli and stimuli held in working memory $[10,28]$. Furthermore, imagery strength correlates positively with visual working memory performance [94,95]. In contrast, a recent study showed that an individual with aphantasia - the inability to generate mental images - was still able to perform a number of working memory tasks [96]. Working memory seems to encompass other cognitive processes besides the generation of mental images, such as the re-coding of visual information into appropriate task responses. Furthermore, imagery is not restricted to working memory, but plays a role in other cognitive processes such as thinking about the future. The exact overlap between mental imagery and working memory remains unclear. In this review we discuss working memory studies as converging evidence to imagery research if they included a clear visual imagery aspect.

Because of the strong relationship between the two, the large neural overlap between perception and imagery reported in this review is in line with the sensory recruitment model of working memory. This theory proposes that the same cortical regions that support perceptual processing of a stimulus are recruited to maintain that information in working memory [97-99]. One argument that has been used against this theory is that working memory content is often represented in fronto-parietal cortex [100]. However, these observations would be in accordance with the sensory recruitment model if those areas are also involved in perceptual processing. The overlap between imagery and perception in fronto-parietal areas reported here supports this idea. Future studies should further investigate the differences and similarities between working memory and imagery to fully understand the implications of the overlap between imagery and perception for theories of working memory.

\section{Neural representations}

Most processing during perception happens in the occipital and temporal lobes. In this part of the cortex, also referred to as the visual cortex, basic visual features are detected and 
merged into coherent percepts. The subjective overlap between perception and imagery is caused by the fact that they lead to the experience of similar visual features: perceiving and imagining a cat both give rise to an experience of pointy ears, whiskers and almond-shaped eyes. In accordance with this intuition, early neuroimaging studies showed that imagery and perception are associated with similar category-specific responses in high-level occipitotemporal cortex $[7,8]$.

Studies using more sensitive multivariate pattern analysis (MVPA) showed that perception and imagery rely on similar neural representations throughout the ventral visual stream [8-13]. There also appears to be a gradient in this overlap, with more similar representations in more high-level visual areas [14] (Fig. 1A). The overlap in low-level visual areas depends on the required visual detail of the task [15] and imagery vividness $[10,14,16]$, such that people who experience more vivid mental imagery show more overlap with perception in retinotopic visual areas (Fig. 1B, also see Box 2).

To convincingly make the point that mental imagery, like perception, uses depictive representations [4], it is not enough to show that imagery activates low-level visual areas in a similar way as perception since the variation in activation between the stimuli that is picked up could be caused by other factors such as attention, reward expectation, coherent shape perception, auditory stimulation or overlap in semantic representations [13]. To exclude these alternative explanations, the activation in early visual cortex during imagery should represent low-level features. Indeed, it was shown that activity is retinotopically organized during imagery $[17,18]$ and that encoding models based on low-level features derived from perception can successfully identify imagined stimuli [13]. Furthermore, behavioral priming effects of imagery on perception (discussed in more detail in the section 'Function of the overlap') are location and orientation specific $[19,20]$, further suggesting that imagery activates low-level perceptual features.

Neuropsychological evidence regarding the effects of specific cortical damage on imagery and perception has been more mixed. For example, one patient experienced both perceptual and imagery deficits after extensive bilateral damage to the temporal poles. However, after the perceptual deficits disappeared, the imagery impairment remained [21]. Furthermore, whereas most patients with unilateral visual field deficits are worse at imagining stimuli on the side of their visual field loss, some patients do not show this associated imagery defect [22]. These findings suggest a dissociation between imagery and 
perception in the ventral visual stream. However, it is difficult to draw firm conclusions from these studies because the damage is heterogeneous between patients and rarely concerns only one specific brain area. In the case of impaired imagery with intact perception, it is possible that brain areas involved in the generation of mental images were also damaged. Moreover, in the case of intact imagery with impaired perception, the damage might have been specific to the input layer of the visual cortex, disrupting bottom-up signals while keeping top-down signals intact $[23,24]$.
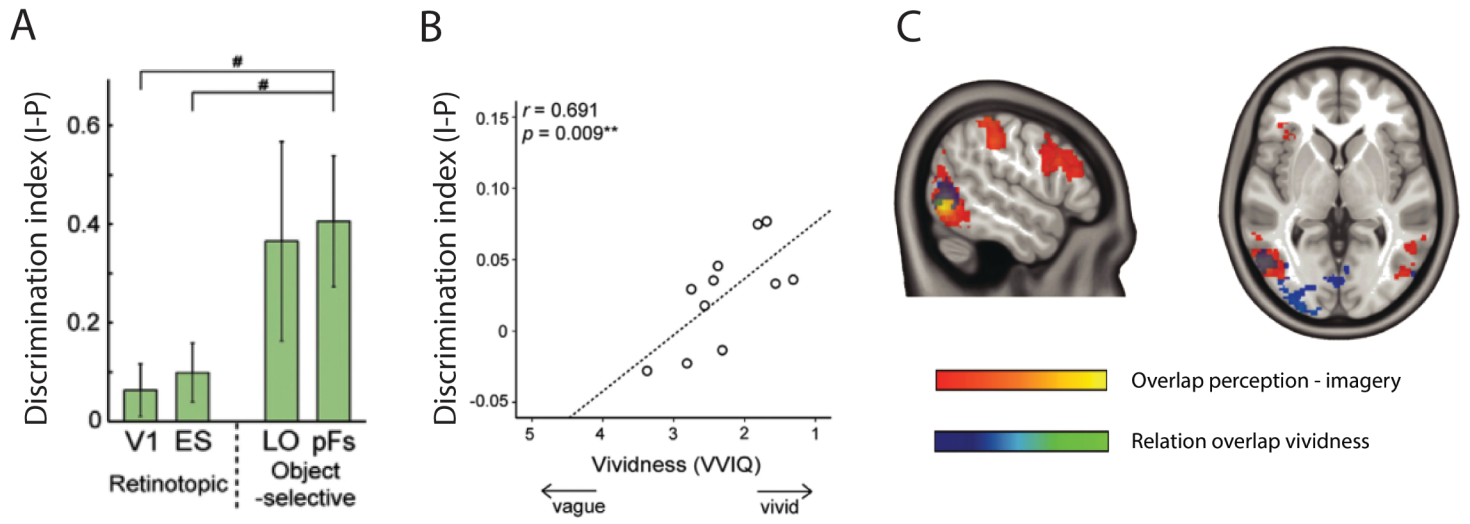

Figure 1. Overlap in neural representations between perception and imagery estimated using fMRI. (A) Adapted from [14]. The overlap in neural representations of imagined and perceived stimuli was assessed using cross-decoding in four different regions of interest along the ventral visual stream. The results indicate that there is more overlap between imagery and perception in high-level, object-selective areas compared to low-level, retinotopic areas. (B) Adapted from [14]. The overlap in retinotopic areas was correlated with participants' imagery vividness scores. The positive correlation shows that people reporting more vivid imagery have higher overlap with perception in these areas. (C) Adapted from [25]. The neural overlap between imagined and perceived stimuli was assessed across the whole brain. Red/yellow indicates significant overlap and blue/green indicates a modulation of the overlap by imagery vividness. There was significant overlap in visual, parietal and frontal areas but only the overlap in visual areas is modulated by imagery vividness.

Most of the studies mentioned above focused on the ventral visual stream. However, a recent study showed that imagery and perception also activate similar neural representations in fronto-parietal areas [25] (Fig 1C). Other studies have shown stimulus information in fronto-parietal cortex during perception $[26,27]$ and working memory $[28,29]$, indicating that these areas contain content representations during both forms of visual experience. As these areas likely do not represent exactly the same information, the question arises what the functions of these different neural representations are for visual experience.

Parietal cortex has been known to play an important role in spatial and feature-based attention in perception [30]. One study showed that also during imagery, focusing on different features of an imagined stimulus increased activity in the intraparietal sulcus (IPS) 
[31]. Furthermore, a recent meta-analysis showed that parietal cortex is most consistently activated in imagery tasks requiring spatial comparisons [32]. Moreover, TMS above the intraparietal sulcus leads to decreased performance during spatial comparisons between mental images [33]. Together, this suggests that parietal cortex is specifically involved in spatial and feature-based attention during both perception and imagery. On the other hand, decoding studies have revealed stimulus representations in parietal cortex during perception, imagery and working memory [25,34]. An explanation for these findings is that parietal cortex encodes which parts of imagined and perceived stimuli are salient $[35,36]$, which can then be used for top-down attention (Fig. 2, Key Figure).

Frontal cortex has also been implied in selective attention during perception as well as imagery $[37,38]$. At the same time, stimulus identity can be decoded from frontal areas during working memory [34] as well as perception [27,39,40]. Furthermore, there is some evidence that the neural representations of imagined and perceived stimuli in frontal areas are similar $[9,25]$. Representations in frontal cortex seem to be strongly influenced by task demands $[27,41,42]$. There is little overlap in stimulus representations between tasks [41] and decoding the task identity leads to much higher accuracies than decoding the stimulus $[27,41,42]$. Therefore, frontal cortex might be involved in representing the task-relevant structure of the stimuli during both perception and imagery (Fig. 2, Key Figure). Future research should further explore neural representations of imagined and perceived stimuli during different tasks to disentangle their function in generating visual experience. 


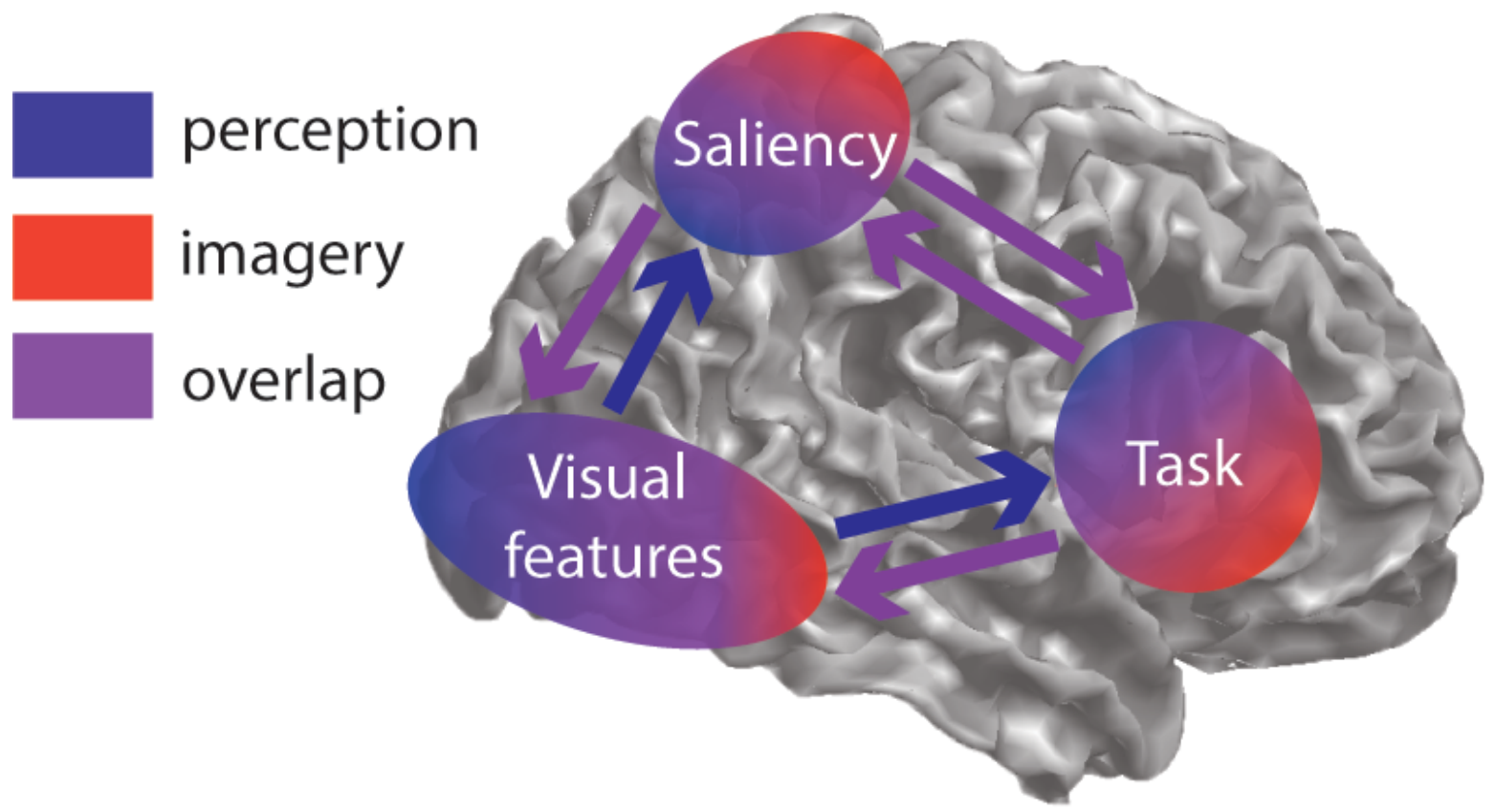

Figure 2, Key Figure. Shared neural mechanisms of visual perception and imagery. Neural representations of imagined and perceived stimuli show overlap in ventral visual areas as well as parietal and frontal areas. During both perception and imagery, visual areas represent the visual features of the sensory experience. Parietal areas encode the saliency, which is used for top-down spatial and featured-based attention during perception as well as during imagery. Representations in frontal areas are more task- than content-dependent, indicating that frontal areas represent task-relevant structure during both imagery and perception. There seems to be large overlap in top-down mechanisms between imagery and perception whereas the bottom-up processing characteristic of perception is absent during imagery.

\section{Temporal dynamics}

How activation progresses through the brain during perception has been studied extensively for many years [43-45]. After light hits the retina, activation reaches the cortex after approximately 50 milliseconds [46]. Then, signals travel towards more anterior visual areas over time where high-level category representations are activated at around 150 milliseconds [47-50]. Recurrent processing is assumed to then further sharpen the visual representation [51-53]. Finally, depending on the exact task, after about 300 milliseconds, activation reaches fronto-parietal areas [54-56]. In contrast to perception, imagery is caused by internal signals from (working) memory. To what extent do imagery and perception rely on similar temporal dynamics?

The dynamics of neural processing can be investigated by looking at the temporal generalization of neural representations, which is inferred by training a classifier on one time point and testing it on other time points, usually using whole-brain EEG or MEG signals [57]. 
An example of such a temporal generalization matrix during perception is shown in Figure $3 \mathrm{~A}$. A diagonal pattern indicates that the classifier can only generalize to neighboring time points which means that the neural representation changes rapidly over time. In contrast, offdiagonal above-chance accuracy indicates that the representation is stable over time or gets reactivated at different times [57]. Temporal generalization during perception generally shows a combination of diagonal and off-diagonal decoding, indicating sequential as well as sustained processing $[47,48,58,59]$ (Fig. 3A).

Recently, the temporal generalization during imagery was investigated [60] (Fig. 3B). The onset of imagery is defined as the onset of a cue that instructs the generation of a mental image. In contrast to perception, during imagery, there was broad off-diagonal generalization from the onset. This is in line with findings on representational stability during working memory $[61,62]$. This could indicate that during imagery there are no clear distinct processing stages, but instead the entire visual representation gets activated at once [57]. However, it is also possible that this strong off-diagonal generalization is caused by jitter in the onset between different trials [63]. Since these analyses require averaging over trials per time point, any time-locked process will be obscured by large timing differences between trials. At this point, it is impossible to confidently rule out this explanation and more research is needed to fully uncover the temporal dynamics during imagery.

Temporal overlap between perception and imagery was also investigated (Fig. 3C). The results indicate that imagery specifically overlaps with perceptual processing around 150 milliseconds and after 300 milliseconds. This overlap around 150 milliseconds found with MEG is in line with the robust fMRI findings on overlap in category representations during perception and imagery $[7,8,50]$. Furthermore, the overlap after 300 milliseconds fits well with the reported overlap in fronto-parietal areas $[25,54-56]$. The absence of early overlap shows that the earliest processing steps of perception do not generalize to imagery. This could be because low-level representations first have to be sharpened by feedback before they are in a format that can be accessed by top-down imagery. Another possibility is that the activation of early perceptual representations during imagery is more transient and variable over time than the activation of late perceptual representations [60]. 
A

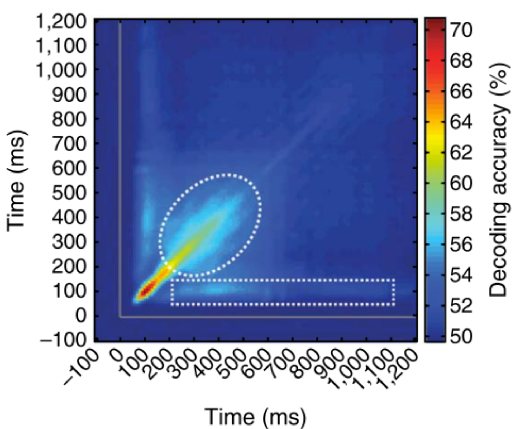

B

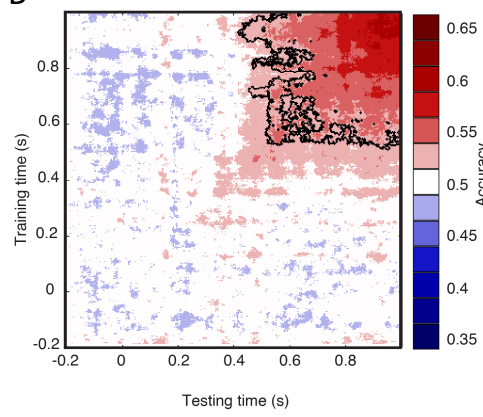

C

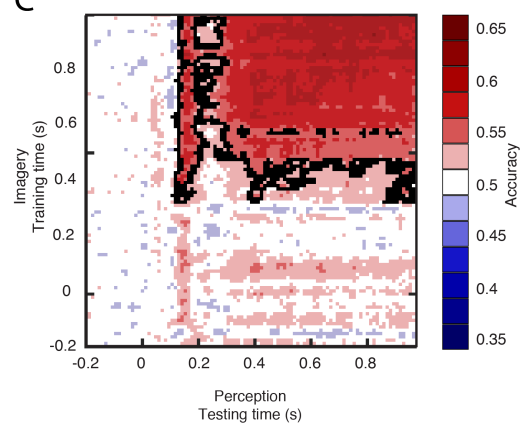

Figure 3. Temporal dynamics during perception and imagery. Temporal generalization matrices: a classifier is trained on a specific time point and then tested on all other time points, providing a measure of how well neural representations at certain time points generalize. The $y$-axis represents the training time and the $x$-axis represents the testing time. The colors show decoding accuracy. (A) Adapted from [52]. Temporal generalization of MEG signals during object perception. The eclipse shows diagonal generalization, indicating rapidly changing representations. The rectangle indicates off-diagonal generalization indicating a stable representation around that training time. (B)-(C) Adapted from [54] (B) Temporal generalization of MEG signals during imagery of faces and houses. The square pattern indicates broad off-diagonal generalization. (C) Temporal generalization of MEG signals between perception and imagery. A classifier was trained at different time points during imagery and tested at different time points during perception. Perceptual processing around $130 \mathrm{~ms}$ and after $300 \mathrm{~ms}$ generalized to imagery.

Furthermore, it should be noted that temporal generalization methods generally rely on scalp EEG and MEG. These methods are well known for their spatial ambiguity: the same scalp distribution could be caused by different underlying neural sources. This makes it hard to draw firm conclusions about overlap in neural representations over time and between conditions. However, recently it has been shown that within-participant decoding, the method underlying temporal generalization, is potentially more sensitive to subtle differences in underlying spatial patterns $[64,65]$. Furthermore, the temporal generalization results reported here are largely in line with electrophysiological and fMRI studies. To conclude, more research is needed to fully understand the temporal dynamics underlying perception and imagery, but the studies presented here provide interesting first ideas. 


\section{Box 2: Vividness of Visual Imagery and the Visual Cortex}

Already in 1880, Francis Galton observed that people vary in the degree to which they can generate vivid mental images [101]. In the last few years, a lot of attention has been given to the case of aphantasia, a term that describes people who completely lack the ability to form mental images [88]. The neural mechanisms of aphantasia are still largely unknown. However, many studies have investigated the neural correlates of imagery vividness.

The activation in visual areas during imagery and especially the overlap of this activation with perception, correlates positively with self-report measures of imagery vividness $[10,14,16,25,102]$ (for more details, see main text). Furthermore, imagery vividness modulates top-down connectivity to early visual areas [74] (Fig. 4). Finally, a recent study [103] reported that the size of V1 was negatively correlated with imagery strength and positively with imagery precision. Taken together, the visual cortex seems to be essential in determining the vividness of internally generated visual experience. This fits well with the idea that this part of the brain is involved in retinotopically representing visual information in a detailed manner during both forms of visual experience.

\section{Directional connectivity}

Developing a detailed understanding of the overlap in neural mechanisms during perception and imagery requires going beyond neural representations to interactions between different neural populations. In one study, dynamic causal modelling (DCM) [66] on fMRI data revealed that the category-specific activation in occipito-temporal cortex could best be explained by bottom-up connections from inferior occipital cortex during perception, and by top-down connections from frontal cortex during imagery [67]. A more recent high-density EEG study found stronger bottom-up coupling between inferior occipital cortex and superior parietal lobe during perception compared to imagery [68].

These studies are in line with the traditional notion of perception as a purely bottomup process and imagery as a top-down process [4]. However, bottom-up processing could theoretically also be involved in imagery. For example, early visual areas might first be 
activated in a top-down manner, followed by a bottom-up propagation of activity. Furthermore, there exists a large body of research showing that top-down processes also play an important role in perception [69-71]. Using DCM on fMRI data [72,73], a recent study showed that during both imagery and perception, there is a strong increase in top-down coupling between inferior frontal gyrus (IFG) and visual cortex compared to baseline [74] (Fig. 4). This increase was much stronger during imagery than during perception. Furthermore, there is an increase in top-down coupling between parietal cortex and visual cortex during perception. This connection was also modulated by imagery vividness. In line with previous studies, the increase in bottom-up connectivity characteristic for perception was absent during imagery [74].

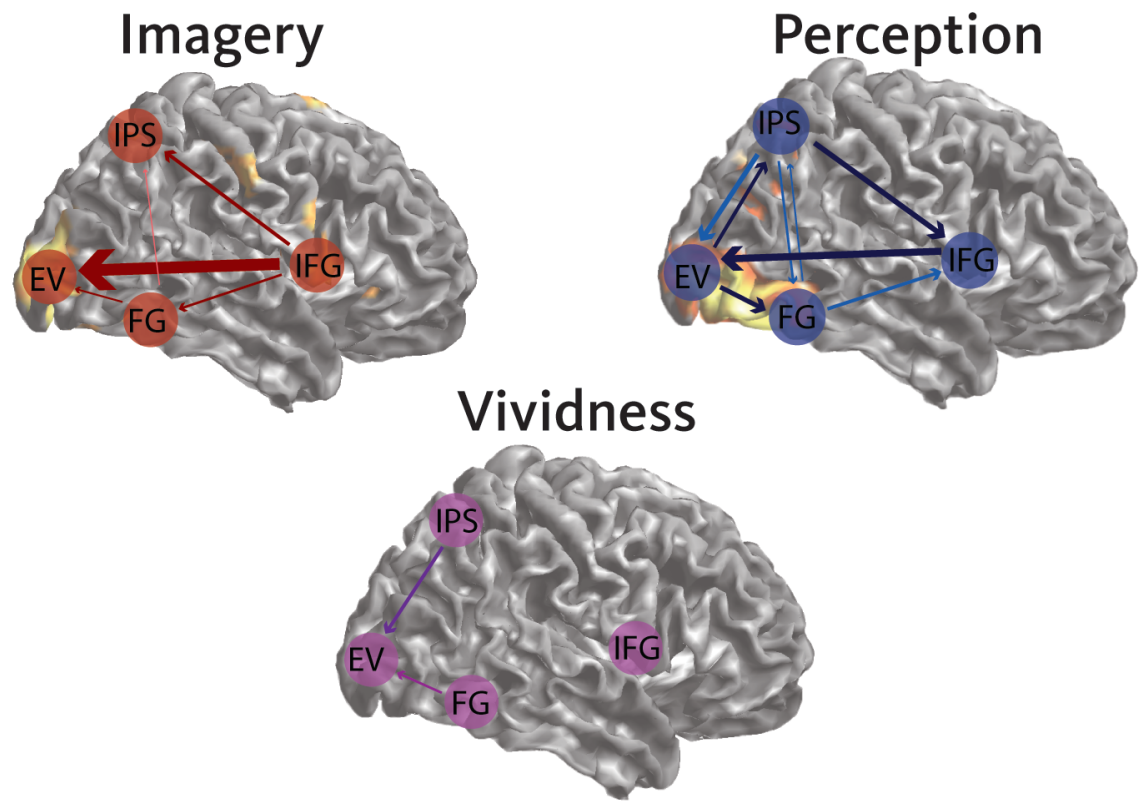

Figure 4. Effective connectivity during perception and imagery. Adapted from Dijkstra et al. (2017b). The results were obtained using Dynamic Causal Modelling (DCM) of $\mathrm{fMRI}$ data during perception and imagery on four ROIs: early visual cortex (EV), high-level visual cortex/fusiform gyrus (FG), intraparietal sulcus (IPS) and inferior frontal gyrus (IFG). The shown arrows reflect connections that were significantly modulated on the group level by the different factors. The width of the arrows indicate the strength of the influence and light arrows reflect inhibitory connections while dark arrows reflect excitatory connections. Both imagery and perception rely on top-down recruitment of visual areas. The vividness of visual imagery correlates with the strength of top-down recruitment of early visual areas. Furthermore, whereas perception is accompanied by bottom-up connectivity, this is absent during imagery.

Top-down coupling between IFG and visual cortex has been proposed to be important for selective attention in the presence of a visual stimulus $[75,76]$, as well as for the maintenance of visual information in the absence of a stimulus $[37,77]$. One hypothesis is that this coupling 
reflects the transformation from more abstract stimulus information into more sensory stimulus representations. During perception, this would result in an attentional template in visual cortex that enhances the response to task-relevant information. During imagery, this coupling is stronger, which might lead to increased activation of these sensory representations and therefore give rise to internally generated visual experience in the absence of bottom-up input. Future studies should investigate which factors modulate the strength of this coupling. Furthermore, in line with the idea that the IPS is involved in spatial and feature-based attention during both forms of visual experience, top-down coupling between IPS and visual cortex was found during perception $[67,74]$, imagery $[67,68]$ and was modulated by imagery vividness [74]. It should be noted that the results of these directional connectivity studies rely to some extent on the assumptions made by the specific models. More studies with different methodologies are needed to ensure the robustness of the results.

\section{Function of the overlap}

The large overlap in neural mechanisms between perception and imagery reported here raises the question to what extent this overlap has functional relevance. One hypothesis is that the overlap does not have a function, but that it is just more efficient to use the same neural mechanisms for similar processes. Alternatively, the large overlap between imagery and perception might reflect an underlying cognitive function.

This is proposed in the emulation theory of mental imagery [78], [79]. This theory posits that mental imagery is a way to simulate what the world would look like in different situations. The emulation part of the theory indicates that it concerns a form of simulation whereby also the processes involved in the simulated event, and not only the event itself, are mimicked [79]. In the case of imagery this means that not only the content/stimulus is simulated but that also the processes involved in the perception of that content are mimicked. This is in line with the large overlap in sensory representations during imagery and perception. In other words, emulation theory states that mental imagery provides a way to generate explicit predictions about sensory input given different situations $[78,79]$. In the context of artificial intelligence, is has been shown that the emulation of sensory events can be used to inform future actions [80]. 
This idea could also explain behavioral results on the influence of imagery on conscious perception during binocular rivalry. Binocular rivalry is the phenomenon that when a different image is presented to each eye, only one of them is consciously perceived, while the other one is suppressed [81]. A series of studies have shown that when one of the two images is imagined prior to the rivalry display, this image has a higher probability of becoming dominant $[19,82,83]$. In other words, imagery can prime conscious perception. These results can be explained by the idea that imagery simulates perception. In this set-up, prior imagery would then pre-activate the perceptual representation of one image, leading to an enhanced response for that image upon congruent sensory input [84]. In line with this idea, it has been shown that imagining a face prior to perceiving a face leads to an enhanced face-specific ERP response [85].

The emulation theory of imagery also states that perception is in part emulation: "The role played by sensation (bottom-up sensory input) is to constrain the configuration and evolution of this (emulated) representation. In motto form, perception is a controlled hallucination process" (Grush, 2004, p. 393). This idea fits very well with predictive coding accounts of perception, stating that perception arises by comparing top-down predictions with bottom-up sensory input $[51,86]$. The high overlap between imagery and perception for top-down mechanisms reported in this review (high-level representations, top-down connectivity) would then suggest that imagery and perception also rely on similar simulation/prediction processes.

However, the case of aphantasia, a condition describing people who are unable to form mental images (see Box 2), argues against such a strong interpretation. People with aphantasia do not report any perceptual deficits, suggesting no issues with simulation per se. In contrast, they do lack the imagery priming reported above [87]. The majority of people with aphantasia do report involuntary imagery, for example during dreams [88]. This suggests a dissociation between the involuntary, automatic simulation underlying perception and dreaming and the deliberate, conscious simulation that underlies imagery $[89,90]$. On the other hand, the large overlap in the top-down mechanisms of perception and imagery presented here suggests that they rely at least on partly similar processes. Future research should focus on further characterizing the overlap and dissociation between these two forms of simulation by utilizing individual differences in imagery ability. 


\section{Box 3: Overlap in other sensory modalities}

A question that arises is to what extent the overlap between imagery and perception reported here for visual experience generalizes to other modalities. There is evidence for recruitment of the corresponding sensory cortex during auditory imagery [104,105], odor imagery $[106,107]$, tactile imagery [108] and motor imagery $[109,110]$. Future research is necessary to fully explore whether all the results in the visual domain generalize to other domains. For example, is there also a low-level to high-level gradient in the overlap, does vividness correlate with the overlap and is there overlap in top-down connectivity?

\section{Concluding Remarks and Future Perspectives}

Taken together, there is convincing evidence that perception and imagery share a variety of neural mechanisms. Neural representations of perceived and imagined stimuli are similar in visual, parietal and frontal cortex. Visual features are represented in the ventral visual stream during both forms of visual experience. The overlap between perception and imagery is higher in more high-level visual areas, but there is significant overlap even in V1. Moreover, the overlap in early visual areas is modulated by imagery vividness. Together with behavioral evidence, this suggests that imagery simulates perceptual events, thereby explicitly predicting sensory effects of different situations.

Furthermore, the parietal cortex seems to be involved in spatial and feature-based attention during both forms of visual experience. On the other hand, frontal areas represent task-relevant stimulus structure in a similar way during perception and imagery. The temporal dynamics during imagery and perception seem to be different, but it is hard to draw firm conclusions due to the temporal uncertainty during imagery. Finally, in contrast to perception, there is no clear bottom-up connectivity during imagery. However, top-down coupling from frontal cortex to visual areas is present during both perception and imagery. These findings are in line with the idea that imagery and perception rely on similar top-down simulation/prediction processes. The fact that it is possible to have imagery deficits without 
associated perceptual issues indicates that the simulation process underlying perception and imagery is not exactly the same.

These findings converge to the idea that our visual experience, regardless of whether it is triggered by external events or internally generated, relies for a large part on a simulation process. This process is initiated automatically during perception and can be utilized voluntarily during imagery. This idea fits well with predictive processing accounts of perception $[51,91]$. The findings mentioned here also indicate an anterior to posterior hierarchy of abstraction, with more abstract task rules represented frontally and concrete sensory details represented in posterior visual areas. Future research should explore the function of each of these representations within this simulation process. It should be investigated what kind of information is communicated between these areas and to what extent this is similar during imagery and perception.

One question that arises with respect to the large overlap between imagery and perception is how the brain knows what is really out there and what is imagined? If only the top-down mechanisms overlap between perception and imagery, this problem might easily be solved by checking for corresponding bottom-up input [24]. However, if top-down signals activate the same neuronal populations as bottom-up signals do, dissociating between real and imaginary sensory content becomes more difficult. To explore this issue further, future research could investigate how neural representations change when people are aware that what they see is not there, such as during voluntary imagery, versus when they are not aware, such as during hallucinations. Uncovering the mechanism responsible for dissociating real and imagined sensory experience will also have important clinical implications [92].

In conclusion, there is a large body of research showing that perception and imagery rely on similar neural mechanisms. Still, a number of major questions remain unsolved (see Outstanding Questions). Future research should explore to what extent these conclusions extend to other modalities (see Box 3). Furthermore, a relatively unexplored issue is which neural mechanism dissociates sensory activity during imagery and perception and ensures that we generally do not confuse the two in daily life. Finally, there is relatively little research on the interaction between imagery and perception. Such studies would provide insight into the functional relevance of the neural overlap between imagery and perception. 


\section{Acknowledgements}

SB and MvG were supported by VIDI grant number 639.072.513 of The Netherlands

Organization for Scientific Research (NOW). ND was supported by institutional funding.

\section{Glossary}

Dynamic causal modelling (DCM): Effective connectivity estimation technique. Per brain region, the expected signal is predicted based on the hypothesized connectivity profile. The model is then inverted to find the parameters that give the best trade-off between model fit and complexity. Parameters are interpreted as being significant if the parameter exceeds zero with $95 \%$ confidence.

Perception: Visual experience where the content reflects and is caused by an afferent physical stimulus.

Mental imagery: Visual experience where the content does not directly relate to any afferent stimulus but is derived from (working) memory.

Multivariate pattern analysis (MVPA): A form of decoding whereby a classifier is trained to dissociate stimuli based on the pattern of activation across multiple voxels (fMRI) or sensors (EEG/MEG). If the classifier can classify a test set above chance, it can be concluded that the pattern of activation represented the stimulus. If it is possible to use a classifier trained in one condition to classify neural activity in another condition, this suggests that similar neural representations are activated in the two conditions.

Temporal generalisation: By training a classifier on the brain activity at one time point, and then testing this classifier on other time points, the temporal stability of neural processing can be inferred. This is usually shown in a testing time by training time generalization matrix (e.g. Fig. 3).

\section{References}

1 Delamillieure, P. et al. (2010) The resting state questionnaire: An introspective questionnaire for evaluation of inner experience during the conscious resting state. 
Brain Res. Bull. 81, 565-573

2 Pylyshyn, Z.W. (1973) What the mind's eye tells the mind's brain: A critique of mental imagery. Psychol. Bull. 80, 1-24

3 Pylyshyn, Z.W. (1981) The imagery debate: Analogue media versus tacit knowledge.

Psychol. Rev. 88, 16-45

$4 \quad$ Kosslyn, S.M. (1980) Image and mind, Harvard University Press.

5 Kosslyn, S.M. (1973) Scanning visual images:Some structural implications. Percept. Psychophys. 14, 90-94

6 Pearson, J. and Kosslyn, S.M. (2015) The heterogeneity of mental representation: Ending the imagery debate. Proc. Natl. Acad. Sci. U. S. A. 112, 10089-92

7 Ishai, A. et al. (2000) Distributed neural systems for the generation of visual images. Neuron 28, 979-990

8 O'Craven, K.M. and Kanwisher, N. (2000) Mental Imagery of Faces and Places Activates Corresponding Stimulus-Specific Brain Regions. J. Cogn. Neurosci. 12, 10131023

9 Stokes, M. et al. (2010) Codes in Visual Cortex during Mental Imagery. J. Neurosci. 29, 1565-1572

10 Albers, A.M. et al. (2013) Shared representations for working memory and mental imagery in early visual cortex. Curr. Biol. 23, 1427-1431

11 Reddy, L. et al. (2010) Reading the mind's eye: Decoding category information during mental imagery. Neuroimage 50, 818-825

12 Johnson, M.R. and Johnson, M.K. (2014) Decoding individual natural scene representations during perception and imagery. Front. Hum. Neurosci. 8, 59

13 Naselaris, T. et al. (2015) A voxel-wise encoding model for early visual areas decodes mental images of remembered scenes. Neuroimage 105, 215-228

14 Lee, S.-H. et al. (2012) Disentangling visual imagery and perception of real-world objects. Neuroimage 59, 4064-73

15 Kosslyn, S.M. and Thompson, W.L. (2003) When is early visual cortex activated during visual mental imagery? Psychol. Bull. 129, 723-746

16 Cui, X. et al. (2007) Vividness of mental imagery: Individual variability can be measured objectively. Vision Res. 47, 474-478

17 Klein, l. et al. (2004) Retinotopic organization of visual mental images as revealed by functional magnetic resonance imaging. Brain Res. Cogn. Brain Res. 22, 26-31

18 Slotnick, S.D. et al. (2005) Visual mental imagery induces retinotopically organized activation of early visual areas. Cereb. Cortex 15, 1570-83

19 Pearson, J. et al. (2008) The functional impact of mental imagery on conscious perception. Curr. Biol. 18, 982-986

20 Bergmann, J. et al. (2015) Smaller Primary Visual Cortex Is Associated with Stronger, but Less Precise Mental Imagery. Cereb. Cortex DOI: 10.1093/cercor/bhv186

21 Sirigu, A. and Duhamel, J.R. (2001) Motor and visual imagery as two complementary but neurally dissociable mental processes. J. Cogn. Neurosci. 13, 910-919

22 Butter, C.M. et al. (1997) Field-specific deficits in visual imagery following hemianopia due to unilateral occipital infarcts. Brain 120, 217-228

23 Roelfsema, P.R. and De Lange, F.P. (2016) Early Visual Cortex as a Multiscale Cognitive Blackboard. DOI: 10.1146/annurev-vision-111815-114443

24 Kok, P. et al. (2016) Selective activation of the deep layers of the human primary visual cortex by top-down feedback. Curr. Biol. 26, 371-376 
25 Dijkstra, N. et al. (2017) Vividness of Visual Imagery Depends on the Neural Overlap with Perception in Visual Areas. J. Neurosci. 37, 1367-1373

26 González-García, C. et al. (2018) Content-specific activity in frontoparietal and default-mode networks during prior-guided visual perception. Elife 7, 1-25

27 Hebart, M.N. et al. (2018) The representational dynamics of task and object processing in humans. Elife 7 ,

28 Christophel, T.B. et al. (2015) Parietal and early visual cortices encode working memory content across mental transformations. Neuroimage 106, 198-206

29 Christophel, T.B. et al. (2017) The Distributed Nature of Working Memory. Trends Cogn. Sci. 21, 111-124

30 Malhotra, P. et al. (2009) Role of right posterior parietal cortex in maintaining attention to spatial locations over time. Brain 132, 645-60

31 Ishai, A. et al. (2002) Visual imagery of famous faces: effects of memory and attention revealed by fMRI. Neuroimage $17,1729-1741$

32 Winlove, C. et al. (2018) The neural correlates of visual imagery: a co-ordinate-based meta-analysis. Cortex DOI: 10.1016/j.cortex.2017.12.014

33 Sack, A.T. et al. (2005) The dynamics of interhemispheric compensatory processes in mental imagery. Science (80-. ). 308, 702-704

34 Christophel, T.B. et al. The Distributed Nature of Working Memory. , Trends in Cognitive Sciences, 21. (2017) , 111-124

35 Bogler, C. et al. (2011) Decoding successive computational stages of saliency processing. Curr. Biol. 21, 1667-71

36 Yun, K. et al. (2013) Exploring the role of gaze behavior and object detection in scene understanding. Front. Psychol. 4, 917

37 Nobre, A.C. et al. (2004) Orienting attention to locations in perceptual versus mental representations. J. Cogn. Neurosci. 16, 363-373

38 Ishai, A. et al. (2002) Visual Imagery of Famous Faces: Effects of Memory and Attention Revealed by fMRI. Neuroimage 17, 1729-1741

39 Jiang, X. et al. (2007) Categorization Training Results in Shape- and Category-Selective Human Neural Plasticity. Neuron 53, 891-903

40 Chan, A.W.-Y. (2013) Functional organization and visual representations of human ventral lateral prefrontal cortex. Front. Psychol. 4, 371

41 Bugatus, L. et al. (2017) Task alters category representations in prefrontal but not high-level visual cortex. Neuroimage DOI: 10.1016/j.neuroimage.2017.03.062

42 Lee, S.-H. et al. (2013) Goal-dependent dissociation of visual and prefrontal cortices during working memory. Nat. Neurosci. 16, 997-9

43 Van Essen, D.C. et al. (1992) Information processing in the primate visual system: an integrated systems perspective. Science (80-. ). 255, 419-23

44 Hubel, D.H. and Wiesel, T.N. (1968) Receptive Fields and Functional Architecture of monkey striate cortex. J. Physiol. 195, 215-243

45 Maunsell, J.H.R. and Newsome, W.T. (1987) Visual Processing in Monkey Extrastriate Cortex. Annu. Rev. Neurosci. 10, 363-401

46 Thorpe, S.J. and Fabre-Thorpe, M. (2001) Seeking categories in the brain. Science 291, 260-3

47 Carlson, T.A. (2011) High temporal resolution decoding of object position and category. J. Vis. 11, 1-17

48 Isik, L. et al. (2014) The dynamics of invariant object recognition in the human visual 
system. J. Neurophysiol. 111, 91-102

49 Seeliger, K. et al. (2017) Convolutional neural network-based encoding and decoding of visual object recognition in space and time. Neuroimage DOI:

10.1016/j.neuroimage.2017.07.018

50 Thorpe, S. et al. Speed of processing in the human visual system. , Nature, 381. (1996) , 520-522

51 Bastos, A.M. et al. Canonical Microcircuits for Predictive Coding. , Neuron, 76. (2012) , 695-711

52 Bastos, A.M. et al. (2015) Visual areas exert feedforward and feedback influences through distinct frequency channels. Neuron 85, 390-401

53 Cauchoix, M. et al. (2014) The neural dynamics of face detection in the wild revealed by MVPA. J. Neurosci. 34, 846-54

54 Anderer, P. et al. (1997) Electrical sources of P300 event-related brain potentials revealed by low resolution electromagnetic tomography. Neuropsychobiology 37, 2027

55 Soltani, M. and Knight, R.T. (2000) Neural Origins of the P300. Crit. Rev. Neurobiol. 14, 26

56 Mulert, C. et al. (2004) The neural basis of the P300 potential. Eur. Arch. Psychiatry Clin. Neurosci. 254, 190-198

57 King, J.R. and Dehaene, S. Characterizing the dynamics of mental representations: The temporal generalization method. , Trends in Cognitive Sciences, 18. (2014) , 203-210

58 Cichy, R.M. et al. (2014) Resolving human object recognition in space and time. Nat. Neurosci. 17,

59 Carlson, T. et al. (2013) Representational dynamics of object vision : The first 1000 ms. J. Vis. 13, 1-19

60 Dijkstra, N. et al. (2018) Differential temporal dynamics during visual imagery and perception. Elife 7, 1-16

61 Spaak, E. et al. (2017) Stable and Dynamic Coding for Working Memory in Primate Prefrontal Cortex. J. Neurosci. 37, 6503-6516

62 King, J.-R. et al. (2016) Brain Mechanisms Underlying the Brief Maintenance of Seen and Unseen Sensory Information. Neuron 92, 1122-1134

63 Vidaurre, D. et al. (2018) Temporally unconstrained decoding reveals consistent but time-varying stages of stimulus processing. bioRxiv DOI: 10.1101/260943

64 Cichy, R.M. et al. (2015) Can visual information encoded in cortical columns be decoded from magnetoencephalography data in humans? Neuroimage 121, 193-204

65 Stokes, M.G. et al. (2015) Decoding Rich Spatial Information with High Temporal Resolution. Trends Cogn. Sci. 19, 636-8

66 Friston, K.J. et al. (2003) Dynamic causal modelling. Neuroimage 19, 1273-1302

67 Mechelli, A. et al. (2004) Where bottom-up meets top-down: neuronal interactions during perception and imagery. Cereb. Cortex 14, 1256-65

68 Dentico, D. et al. (2014) Reversal of cortical information flow during visual imagery as compared to visual perception. Neuroimage 100, 237-43

69 Buschman, T.J. and Miller, E.K. (2007) Top-down versus bottom-up control of attention in the prefrontal and posterior parietal cortices. Science 315, 1860-1862

70 Muckli, L. and Petro, L.S. Network interactions: Non-geniculate input to V1. , Current Opinion in Neurobiology, 23. (2013) , 195-201

71 Muckli, L. (2010) What are we missing here? Brain imaging evidence for higher 
cognitive functions in primary visual cortex V1. Int. J. Imaging Syst. Technol. 20, 131139

72 Friston, K. et al. (2015) Empirical Bayes for DCM: A Group Inversion Scheme. Front. Syst. Neurosci. 9,

73 Friston, K.J. et al. (2016) Bayesian model reduction and empirical Bayes for group (DCM) studies. Neuroimage 128, 413-431

74 Dijkstra, N. et al. (2017) Distinct Top-down and Bottom-up Brain Connectivity during Visual Perception and Imagery. Sci. Rep. 7,

75 Mayer, J.S. et al. (2007) Common neural substrates for visual working memory and attention. Neuroimage 36, 441-453

76 Zanto, T.P. et al. (2011) Causal role of the prefrontal cortex in top-down modulation of visual processing and working memory. Nat. Neurosci. 14, 656-61

77 Higo, T. et al. (2011) Distributed and causal influence of frontal operculum in task control. Proc. Natl. Acad. Sci. U. S. A. 108, 4230-4235

78 Grush, R. (2004) The emulation theory of representation: Motor control, imagery, and perception. Behav. Brain Sci. 27, 377-442

79 Moulton, S.T. and Kosslyn, S.M. (2009) Imagining predictions: Mental imagery as mental emulation. Philos. Trans. R. Soc. B Biol. Sci. 364, 1273-1280

80 Eslami, S.M.A. et al. (2018) Neural scene representation and rendering. Science 360, 1204-1210

81 LEVELT, W.J.M. (1966) THE ALTERNATION PROCESS IN BINOCULAR RIVALRY. Br. J. Psychol. 57, 225-238

82 Sherwood, R. and Pearson, J. (2010) Closing the mind's eye: incoming luminance signals disrupt visual imagery. PLoS One 5, e15217

83 Keogh, R. and Pearson, J. (2014) The sensory strength of voluntary visual imagery predicts visual working memory capacity. J. Vis. 14, 1427-1431

84 Kok, P. et al. (2017) Prior expectations induce prestimulus sensory templates. Proc. Natl. Acad. Sci. DOI: 10.1073/pnas.1705652114

85 Ganis, G. and Schendan, H.E. (2008) Visual mental imagery and perception produce opposite adaptation effects on early brain potentials. Neuroimage 42, 1714-1727

86 Mumford, D. (1992) On the computational architecture of the neocortex - II The role of cortico-cortical loops. Biol. Cybern. 66, 241-251

87 Keogh, R. and Pearson, J. 28-Oct-(2017), The blind mind: No sensory visual imagery in aphantasia. , Cortex, Elsevier

88 Zeman, A. et al. (2015) Lives without imagery e Congenital aphantasia Adam. Cortex 73, 378-380

89 Barsalou, L.W. (2008) Grounded Cognition. Annu. Rev. Psychol. 59, 617-645

90 Kent, C. and Lamberts, K. (2008) The encoding-retrieval relationship: retrieval as mental simulation. Trends Cogn. Sci. 12, 92-98

91 Friston, K. (2005) A theory of cortical responses. Philos. Trans. R. Soc. London B Biol. Sci. 360,

92 Pearson, J. et al. (2015) Mental Imagery: Functional Mechanisms and Clinical Applications. Trends Cogn. Sci. 19, 590-602

93 Tong, F. (2013) Imagery and visual working memory: one and the same? Trends Cogn. Sci. 17, 489-90

94 Keogh, R. and Pearson, J. (2011) Mental imagery and visual working memory. PLoS One 6, e29221 
95 Baddeley, A.D. and Andrade, J. (2000) Working memory and the vividness of imagery. J. Exp. Psychol. Gen. 129, 126-145

96 Jacobs, C. et al. (2017) Visual working memory performance in aphantasia. Cortex DOI: 10.1016/j.cortex.2017.10.014

97 Scimeca, J.M. et al. (2018) Reaffirming the Sensory Recruitment Account of Working Memory. Trends Cogn. Sci. 22, 190-192

98 D’Esposito, M. and Postle, B.R. (2015) The Cognitive Neuroscience of Working Memory, 66

99 D'Esposito, M. (2007) , From cognitive to neural models of working memory. , in Philosophical Transactions of the Royal Society B: Biological Sciences, 362, pp. 761772

100 Xu, Y. 01-Oct-(2017), Reevaluating the Sensory Account of Visual Working Memory Storage. , Trends in Cognitive Sciences, 21, Elsevier Current Trends, 794-815

101 Galton, F. (1880) Statistics of mental imagery. Mind os-V, 301-318

102 Fulford, J. et al. (2017) The neural correlates of visual imagery vividness - an fMRI study and literature review. Cortex DOI: 10.1016/j.cortex.2017.09.014

103 Bergmann, J. et al. (2015) Smaller Primary Visual Cortex Is Associated with Stronger, but Less Precise Mental Imagery. Cereb. Cortex DOI: 10.1093/cercor/bhv186

104 Zatorre, R.J. et al. (2007) When the brain plays music: auditory-motor interactions in music perception and production. Nat. Rev. Neurosci. 8, 547-558

105 Zatorre, R.J. and Halpern, A.R. Mental concerts: Musical imagery and auditory cortex. , Neuron, 47. (2005) , 9-12

106 Djordjevic, J. et al. (2005) Functional neuroimaging of odor imagery. Neuroimage 24, 791-801

107 Djordjevic, J. et al. (2004) The Mind's Nose. Psychol. Sci. 15, 143-148

108 Yoo, S.-S. et al. (1990) Neural substrates of tactile imagery_a functional MRI study _ NeuroReport. Neuroreport at <https://oce.ovid.com/article/00001756-20030324000011/HTML>

109 Porro, C.A. et al. (1996) Primary Motor and Sensory Cortex Activation during Motor Performance and Motor Imagery: A Functional Magnetic Resonance Imaging Study,

110 Dechent, P. et al. (2004) Is the human primary motor cortex involved in motor imagery? Cogn. Brain Res. 19, 138-144 